\title{
Antimatter-Driven Fusion Propulsion Scheme for Solar System Exploration
}

\author{
T. Kammash* and D. L. Galbraith \\ University of Michigan, Ann Arbor, Michigan 48109
}

\begin{abstract}
The potential use of the proton-antiproton annihilation reaction as a driver for an inertially confined, magnetically insulated fusion plasma with application to advanced space propulsion is examined. The fusion scheme utilized is the magnetically insulated inertial confinement fusion (MICF) concept which combines the favorable aspects of both inertial and magnetic fusions into one. Using an appropriate set of governing equations for the fusion plasma, along with those that detail the annihilation reactions and the energy deposition by the annihilation products including contributions from muon catalysis, we calculate the energy gain for the system as well as the amount of antihydrogen needed to ignite the plasma. We find about $13 \mathrm{ng}$ of antihydrogen are needed to supply a megajoule of energy to the plasma, and about $10 \mathrm{~g}$ will be needed for a 220 -mT space vehicle to make a one-way trip to Mars in about 2 months.
\end{abstract}

\section{Introduction}

$\mathbf{I}^{\mathrm{N}}$ $\mathrm{N}$ several previous publications ${ }^{1-3}$ we examined the potential use of the magnetically insulated inertial confinement fusion (MICF) concept as a propulsion device that could be utilized in solar explorations and/or interplanetary travel. The concept in question combines the favorable aspects of both magnetic and inertial fusions in that physical containment of the hot plasma is provided by a metallic shell while its thermal energy is insulated from the material wall by a strong, self-generated magnetic field as illustrated in Fig. 1. Unlike the conventional implosion-type inertial fusion schemes, energy production in this approach does not require compression of the fusion fuel to many times solid-state densities and simultaneous delivery of energy to the core to initiate the burn. Instead the fusion plasma is created through wall ablation by an incident laser beam that enters the target through a hole. The same laser gives rise to the strong magnetic field through a process known as the "thermoelectric" effect, whereby it can be shown that such a field can be generated in a hot plasma when its density gradient is perpendicular to its temperature gradient. We have seen that MICF is capable of producing specific impulses of several thousand seconds and thrusts of tens of kilonewtons that would allow round trips to Mars, for example, to be made in relatively short periods (a few months) even when the massive power supply system for the laser driver is carried on board. It is clear that such travel times can be substantially reduced if the power supply component is eliminated from the dry weight of the vehicle. Several recent studies ${ }^{4}$ have proposed beaming the needed energy from an Earth-orbiting space station, but it is evident that a more reliable performance can be assured if a compact energy source is taken along. Clearly no source can match that of matter-antimatter annihilation reactions since such a fuel (e.g., anti-hydrogen) possesses the largest specific energy, i.e., energy per unit mass, provided, of course, that the technology for producing, storing, and manipulating such

Presented as Paper 90-2509 at the AIAA/SAE/ASME/ASEE 26th Joint Propulsion Conference, Orlando, FL, July 16-18, 1990; received Sept. 10, 1990; revision received April 12, 1991; accepted for publication April 15, 1991. Copyright @ 1990 by the American Institute of Aeronautics and Astronautics, Inc. All rights reserved.

*Professor, Department of Nuclear Engineering. Senior Member AIAA.

$\dagger$ Assistant Research Scientist, Department of Nuclear Engineering. a fuel can be economically developed. The potential use of antimatter annihilation reactions for advanced propulsion has been the object of many studies in recent years. A comprehensive review of the conceptual designs of such systems has been provided by Cassenti ${ }^{5}$ and the reader is encouraged to consult this reference for details. In this paper we examine the use of the annihilation reaction of protons-antiprotons as a driver (replacing the laser) for an MICF propulsion device and estimate, as an example, the amount needed to undertake a round trip to Mars. A comparison with a laser-driven system for deuterium-tritium (DT) fuel, and deuterium-helium 3 $\left(\mathrm{DHe}^{3}\right)$ fuel will be attempted in order to highlight the performance features of each approach.

\section{Fusion Energy Production in MICF}

The propulsive capability of MICF depends critically on its ability to sustain an ignited fusion plasma, and the generation of large amounts of energy typified by large $Q$ values, where $Q$ is the ratio of fusion energy to the input energy $E_{\text {in }}$. This performance is assessed by solving coupled sets of particle and energy balance equations for each species in the hot core, in the halo, and in the metal shell. ${ }^{6}$ These equations are quasione-dimensional, time-dependent equations in which the plasma components in each region are treated as an ideal gas subject to the adiabatic laws of thermodynamics. For a system that burns a mixture of deuterium-tritium (DT) fuel, the reaction products consist of alpha particles $\left(\mathrm{He}^{4}\right)$ at $3.5 \mathrm{MeV}$ energy and neutrons at $14.1 \mathrm{MeV}$. The neutrons leave the reaction region and the pellet almost instantly, while the alpha particles deposit a significant portion of their energy in the hot plasma, thus keeping it hot and producing more fusion energy. Unless converted to electric power, the neutron energy must ultimately be disposed of through radiators (or other schemes), and even if used to generate electric power, the thermal conversion efficiency is typically $40 \%$ or less and hence the waste heat must somehow be also rejected. Since neutrons can give rise to radioactivity aboard the spacecraft, as well as interfere seriously with the functioning of electronic and navigational equipment, substantial amounts of shielding to protect the crew and equipment will be needed, and that could significantly add to the dry weight of the vehicle. For that reason it might be useful to consider non-neutron-producing fusion reactions such as the deuterium-helium $3\left(\mathrm{DHe}^{3}\right)$ fuel cycle which generates many fewer neutrons (due to DD and DT satellite reactions) but which requires much higher temperature, and correspondingly higher input energy than the DT 


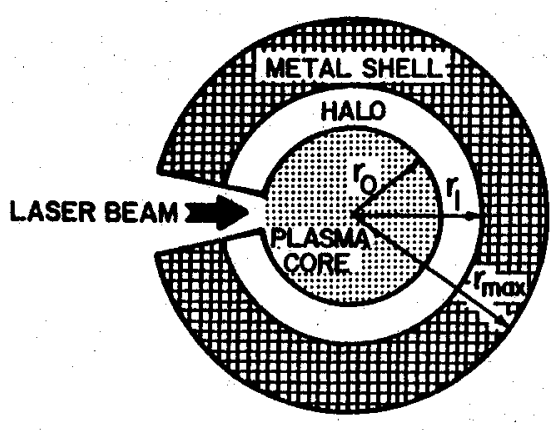

a)

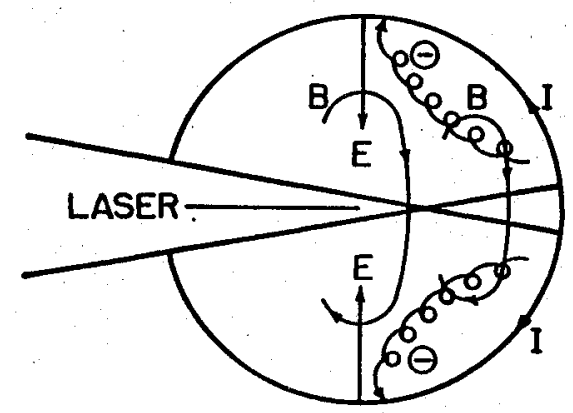

b)

Fig. 1 Schematic diagram of a) plasma formation and b) magnetic field formation in MICF.

fuel cycle, to ignite. We shall examine the performance of MICF for both of these fuel cycles shortly.

The full set of conservation equations alluded to earlier can be found in Ref. 6 . For the sake of illustration we reproduce here the particle and energy balance equations for the fuel ions (DT) which we represent by one species of an average mass of 2.5 atomic mass units (amu) and a density of $n_{f} \mathrm{~cm}^{-3}$. The particle and energy conservation equations are given by

$$
\begin{aligned}
\frac{\mathrm{d}}{\mathrm{d} t} & \left\{\frac{4}{3} \pi r^{3} n_{f}\right\}=-\frac{4}{3} \pi r^{3}\left\{\frac{1}{2} n_{f}^{2}\langle\sigma v\rangle_{f}\right\}+4 \pi r^{2}\left\{\Gamma_{r}-\Gamma_{f}\right\} \\
\frac{\mathrm{d}}{\mathrm{d} t} & \left\{\frac{\frac{4}{3} \pi r^{3} n_{f} T_{f}}{\gamma-1}\right\}+4 \pi r^{2} n_{f} T_{f} \frac{\mathrm{d} r}{\mathrm{~d} t}=\frac{4}{3} \pi r^{3} \\
& \left\{\frac{3}{2} \frac{n_{f} T_{f}}{(n \tau)_{\mathrm{ef}}}\left(T_{e}-T_{f}\right)\right. \\
& +\frac{3}{2} \frac{n_{f} n_{\alpha}}{2(n \tau)_{\alpha f}}\left(T_{\alpha}-T_{f}\right)+n_{f} \sum_{k=1}^{k_{\max }} n_{k}\left(\frac{\mathrm{d} E_{k}}{\mathrm{~d} t}\right)_{f} \\
& \left.-\frac{3}{4} n_{f}^{2} T_{f}\langle\sigma v\rangle_{f}\right\}+4 \pi r^{2}\left\{W_{r}-W_{f}\right\}
\end{aligned}
$$

In these equations $n_{f}$ denotes the ion density, $\langle\sigma v\rangle_{f}$ the velocity-averaged fusion reaction cross section which is temperature dependent, $\Gamma_{r}$ the particle flux for the refueling ions which cross the magnetic field from the halo region to the core plasma (see Fig. 1), and $\Gamma_{f}$ the flux in the opposite direction. Although not too well understood, the topology of the magnetic field in MICF is assumed to be spherically symmetric consistent with the point model represented by the above equations. The first term on the right-hand side of Eq. (1) reflects the loss of ions due to the fusion reaction while the second term represents the source or the net particle flux entering the core. In the second equation $T_{f}$ denotes the ion temperature and the whole left-hand side represents the change in the plasma thermal energy including the spherical expansion term, with $\gamma=\frac{5}{3}$ being the familiar adiabatic constant. The first term on the right-hand side denotes the energy exchange between the electrons and fuel ions and is characterized by the energy exchange constant ${ }^{7}(n \tau)_{\text {ef }}$, the second term represents the exchange with the thermal alpha particles, while the third term reflects the rate at which the $k$ th fast alpha group loses energy to the fuel ions. The fourth term denotes the energy removed from the fuel ion population due to participation in the fusion reactions, while the last term represents the net energy flux between the refueling ions and ions escaping from the core. Similar equations are used for the other species in the core, i.e., the electrons, thermal alphas, and fast alphas, and for the species that occupy the various regions of the pellet. For the purposes of this calculation it was assumed that the fast alpha particles remain totally in the hot core and not diffuse across the magnetic field into the halo or adjacent regions. This is generally justified on the premise that the thermalization time for these particles is much shorter than their diffusion time. This is not entirely true, however, since the range and gyroradius of an average fast alpha particle may be comparable to the dimensions of the regions in question, and thus it could deposit a significant fraction of its energy outside the core. Preliminary investigation ${ }^{8}$ of this problem reveals that even though less than half of such a particle's energy is deposited in the hot core, the gain factor $Q$ is higher in this case due to the increased pressure in the halo and metallic regions that results in further retardation of expansion of the hot core. In view of this, the results presented in the examples to be discussed as applications may be somewhat conservative.

The energy balance equation for the hot electrons in the core contain, in addition to the terms shown in Eq. (2), radiation terms representing the bremsstrahlung and synchrotron emissions. On the basis of relativistic formulation of the problem $^{9}$ it can be shown that the synchrotron radiative loss over the length of the burn is a very small fraction of the total fusion energy produced and can be safely neglected. Most of the bremsstrahlung radiation emanating from the hot plasma gets absorbed in the outer portion of the halo and the inner surface of the metal shell. This radiation helps sustain the partial ionization that takes place in these regions, and contributes to the heating of the resulting plasma, thereby increasing its pressure. This increased pressure plays an especially important role in that it leads to the compression of the hot plasma core, and an increase in its fusion energy production that arises from the corresponding increase in the fuel ion density.

When the appropriate set of equations are solved it can be shown ${ }^{6}$ that very large $Q$ values can be obtained for moderate input laser energies. This arises from the long plasma confinement in MICF which is dictated by the shock speed in the shell rather than by the sound speed in the plasma itself as is the case in implosion-type approaches. This makes MICF a very attractive energy source for terrestrial powerplants and for potential use as an advanced propulsion system. The energetic charged particles produced by the fusion reactions can generate very large specific impulses and thrusts if, at the end of the burn cycle, they are trapped and exhausted by a magnetic nozzle. These propulsive characteristics of MICF will be examined in conjunction with the examples on Mars missions to be presented later.

\section{Antimatter Annihilation as MICF Driver}

As will be noted shortly, laser input energies of few megajoules will be required to generate the desirable propulsion parameters from MICF. The power supply system along with the radiators and energy handling equipment for lasers with such energy outputs can be truly massive and have a strong impact on travel times. In the interest of safe space travel and physical welfare of the crew it is clear that such journeys 
should be carried out in the shortest possible periods. A significant reduction can be achieved if some of the massive components can be eliminated and a minimum amount of propellant is required. Both of these objectives can be achieved if antimatter annihilation reactions are utilized as the driver for MICF in place of the laser.

It is well known that the proton $(p)$-antiproton $(\bar{p})$ annihilation reaction produces more energy per unit-mass $(9 \times$ $10^{16} \mathrm{~J} / \mathrm{kg}$ ) than all the other fuels considered suitable for propulsion purposes. To be useful in this regard the antiproton should be combined with positrons to form antihydrogen, $\bar{H}$, that someday can be made in the form of ice pellets which can be stored and carried on board. The whole world production of $\bar{p}$ is extremely small and the technology for making $\bar{H}$ is in its infancy if it exists at all, but it is hoped that such technology will develop, and sufficient amount of $\bar{H}$ will be made that will make its utilization for space propulsion possible in the time frame projected for space exploration in the next century. In this section we will examine how such a fuel can be used to drive the MICF fusion plasma described earlier.

When a particle meets its antiparticle they annihilate and the energy equivalent to their total mass, $2 \mathrm{MC}^{2}$, is converted into various new particles and kinetic energy. In the case of proton-antiproton annihilation, three charged and two neutral pions are produced on the average, and each neutral pion quickly decays into two photons. This annihilation process is illustrated by the equation

$$
p+\bar{p} \rightarrow 3 \pi^{ \pm}+2 \pi^{\circ} \rightarrow 3 \pi^{ \pm}+4 \gamma
$$

where the kinetic energy of the products is given by $E_{ \pm}=$ $236 \mathrm{MeV}$, and $E_{\gamma}=187 \mathrm{MeV}$. When the rest mass energies of the pions are added to these kinetic energies, a total of $1876 \mathrm{MeV}$ is obtained, which is equal to the rest mass energies of the initial interacting particles. Thus when $\bar{p}$ annihilates on a hydrogen plasma, essentially all the annihilation energy appears in the form of the energetic pions and photons. At solid hydrogen densities the mean free path of the $187-\mathrm{MeV}$ photons is about $25 \mathrm{~m}$, so that for dimensions representative of the MICF system, these photons will not lose their energy in the plasma. The pions, on the other hand, will lose energy through multiple Coulomb interactions with the electrons at a rate approximately given by Bethe's formula: $\mathrm{d} E / \mathrm{d} x=0.52$ $\mathrm{MeV} / \mathrm{cm}$ in solid hydrogen or DT.

In order to estimate the amount of $\bar{H}$ needed to ignite the plasma in MICF, we assume that a tiny pellet of $H$ ice, of radius $R_{s}$, is injected into the target pellet through the hole that normally provides the entry for the laser beam. When this antimatter comes in contact with the solid DT fuel that coats the inner surface of the pellet, annihilation takes place primarily in the boundary layer in which particles and antiparticles become mixed. Following the analysis suggested in Ref. 10, we assume first that whenever an antiparticle penetrates into the boundary layer it instantly annihilates, and the annihilation rate per unit area is given by the total number of antiparticles impinging on that surface. With the thickness of the boundary layer considered very small, an antiparticle is apt to reach the surface without suffering a collision with another such particle, and if these particles have a Maxwellian distribution, $f_{(v)}$, the flux of antiparticles reaching the surface can be written as

$$
J=\frac{1}{4 \pi} \int \bar{n} v \cdot \mathrm{d} \Omega f(v)=\frac{\bar{n}}{\sqrt{2 \pi}}\left(\frac{T}{M}\right)^{1 / 2}
$$

where

$$
f(v)=\frac{4}{\pi}\left(\frac{M}{2 \pi T}\right)^{3 / 2} v^{2} e^{-M v^{2} / 2 T}
$$

and $\bar{n}$ is the number density of the antiparticles. If we denote by $\bar{N}$ the total number of antiparticles in the $\bar{H}$ ice pellet, i.e.,

$$
\bar{N}=\frac{4}{3} \pi R_{S}^{3} \bar{n}
$$

then it is clear from Eq. (4) and the continuity equation, namely

$$
\frac{\partial \bar{n}}{\partial t}+\nabla \cdot J=0
$$

that

$$
\begin{aligned}
\frac{\mathrm{d} \bar{N}}{\mathrm{~d} t} & =-\frac{\bar{N} C}{\frac{4}{3} \pi R_{s}^{3}}\left[\frac{T}{2 \pi M C^{2}}\right]^{1 / 2} 4 \pi R_{\mathrm{s}}^{2} \\
& =-\frac{3 \bar{N} C}{R_{s}}\left[\frac{T}{2 \pi\left(M C^{2}\right)}\right]^{1 / 2}
\end{aligned}
$$

The pions resulting from the $p \bar{p}$ reactions in the DT boundary layer will heat the $\bar{H}$ pellet, so that the increase in the $\dot{H}$ plasma thermal energy can be expressed by

$$
\mathrm{d} W=3 \bar{N} \mathrm{~d} T=-\mathrm{d} \bar{N}\left[\nu \frac{\mathrm{d} E}{\mathrm{~d} x}+\frac{\varepsilon}{\lambda}\right]\langle r\rangle \frac{\bar{N}}{N_{0}}
$$

where $\nu=3$ is the number of charged pions, $\varepsilon$ is the local energy deposition by the recoiling nucleus and the various pion-nucleus interaction debris, $\lambda=3 \mathrm{~cm}$ is the approximate range of the $20-\mathrm{MeV}$ recoil protons from $\bar{p}$ annihilation in $\mathrm{DT}$, and $\langle r\rangle$ is the mean distance traversed by these particles in the $\bar{H}$ pellet whose radius is $R_{s}$. The initial value of $\bar{N}$ is $N_{0}$, and for $\bar{p}$ annihilation in DT the quantity $\varepsilon$ is about 12 $\mathrm{MeV}$. From simple geometric considerations it can be shown that

$$
\langle r\rangle=\frac{4 R_{s}}{\pi}
$$

so that Eq. (7) can be put in the form

$$
\frac{\mathrm{d} W}{\mathrm{~d} t}=3 \bar{N} \frac{\mathrm{d} T}{\mathrm{~d} t}=-\frac{\mathrm{d} \bar{N}}{\mathrm{~d} t}\left\{\frac{1}{2}\left[\nu \frac{\mathrm{d} E}{\mathrm{~d} x}+\frac{\varepsilon}{\lambda}\right] \frac{4 R_{s}}{\pi}\right\} \frac{\bar{N}}{N_{0}}
$$

which must be solved in conjunction with Eq. (6) to produce the time evolution of $\bar{N}$, and the temperature of the antimatter plasma. The solutions can be shown to be

$$
\begin{gathered}
T=T_{m} \tanh ^{2}\left(t / \tau_{a}\right) \\
\bar{N}=N_{\mathrm{o}}\left[1-\tanh ^{2}\left(t / \tau_{a}\right)\right]
\end{gathered}
$$

where $T_{m}$ and the annihilation time $\tau_{a}$ are given by

$$
\begin{gathered}
T_{m}=\frac{2 R_{s}}{3 \pi}\left[\nu \frac{\mathrm{d} E}{\mathrm{~d} x}+\frac{\varepsilon}{\lambda}\right] \\
\tau_{a}=\frac{2 R_{s}}{3 C T_{m}^{1 / 2}}\left[2 \pi\left(M C^{2}\right)\right]^{1 / 2}
\end{gathered}
$$

with $M C^{2}$ being the rest mass energy of $\bar{p}$. Using the previously stated values for the parameters in the above equations we find that

$$
\begin{gathered}
T_{m}=\left(1.180 \times 10^{3}\right) R_{s} \\
\tau_{a}=\left(5.399 \times 10^{-8}\right) \frac{R_{s}}{T_{m}^{1 / 2}}=1.5717 \times 10^{-9} R_{s}^{1 / 2}
\end{gathered}
$$


In order to apply these results to the ignition of MICF we utilize what might be described as the "spark model" whereby a tiny spherical pellet of antihydrogen is suddenly placed at the center of the DT fuel in MICF whose radius is $R_{f}$. The pions generated by the annihilation of the $\bar{H}$ pellet along with the recoil energy will proceed to heat the DT fuel which we treat as a plasma with density $n_{f}$, temperature $T_{f}$ in a sphere of radius $R_{f}$. If we now denote by $E_{f}$ the total energy needed in the fusion fuel, namely

$$
E_{f}=3 n_{f} T_{f}\left(\frac{4}{3} \pi R_{f}^{3}\right)
$$

then we could relate this to the energy absorbed from the annihilation process, using the stopping power given in Eq. (7) and assuming perfect coupling. The result is

$$
E_{f}=\frac{4}{3} \pi R_{s}^{3} n_{s}\left[\nu \frac{\mathrm{d} E}{\mathrm{~d} x} R_{f}+\varepsilon\right]
$$

or approximately given by

$$
E_{f} \cong \frac{4}{3} \pi R_{s}^{3} n_{s}[\varepsilon]
$$

With $n_{s}$ being the spark density $(=\bar{n})$ which we take to be $5 \times 10^{22}$ corresponding to liquid hydrogen density of 0.083 $\mathrm{g} / \mathrm{cm}^{3}$. If (as we shall note shortly) $E_{f}=1.6 \mathrm{MJ}$ as required by the performance of MICF through the parameters $n_{f}=5$ $\times 10^{21}, R_{f}=0.25, T_{f}=10 \mathrm{keV}$, then it is clear from the above equation that the radius of the $\bar{H}$ sphere must be $R_{s}$ $=0.01575 \mathrm{~cm}$, which is much smaller than $R_{f}$, thereby justifying the concept of a tiny "spark." Substituting this value of $R_{s}$ in Eqs. (13) and (14) we find $T_{m}=18.59 \mathrm{keV}$ and $\tau_{a}$ $=1.972 \times 10^{-10} \mathrm{~s} \cong 0.2 \mathrm{~ns}$. The number of $\bar{H}$ particles needed to provide the described energy is simply

$$
\vec{N}=\frac{4}{3} \pi R_{s}^{3} n_{s}=8.181 \times 10^{17}
$$

or in grams:

$$
\bar{\rho}=\bar{N} M=8.181 \times 10^{17} \times 1.66 \times 10^{-24}=1.358 \mu \mathrm{g}
$$

The above result does not take into account the fusion reaction enhancement due to muon catalysis. It should be noted that the charged pions decay into muons $(\mu)$ and neutrinos $\left(\nu_{\mu}\right)$ in accordance with the relations

$$
\begin{aligned}
& \mathrm{II}^{+} \rightarrow \mu^{+}+\nu_{\mu} \\
& \Pi^{-} \rightarrow \mu^{-}+\bar{\nu}_{\mu}
\end{aligned}
$$

keeping in mind that the muons are effectively heavy electrons (207 times the electron mass) and the neutrinos are considered massless. Of special relevance to the problem at hand is the fact that the charged muons have a mean life of $2.2 \times 10^{-6}$ $\mathrm{s}$ which makes their utilization in the present propulsion scheme quite effective.

It has been known for some time ${ }^{11}$ that muons introduced in relatively cold, dense deuterium-tritium (DT) mixture can replace the atomic electrons and form muonic molecules which participate readily in nuclear fusion reactions. Catalysis yields of about 150 fusions per muon, which have exceeded theoretical expectations, have been achieved. The sequence of events in this process can be illustrated by the following chain. A negative muon enters the DT mixture $(\mu+D+T)$, replaces the electron to form a $(T \mu)$ atom, which combines with $D$ to form a muonic molecule (DT $\mu$ ) which in turn leads to a DT fusion reaction freeing the muon to repeat the cycle. Occasionally the muon is captured by the alpha particle $(\alpha)$ following the fusion reaction so that the particles at that stage $(\alpha+\mu+n)$ combine to become $(\alpha \mu+n)$ where the neutron $(n)$ remains free and carries most of the energy produced by the reaction, i.e., 14.1 MeV out of $17.6 \mathrm{MeV}$. Muon retention by the alpha particle, the so-called sticking coefficient, though not fully determined, is believed to be less than $0.4 \%$ and as a result the muon-catalyzed DT fusion cycle can easily be repeated more than 100 times.

To make sure that muon catalysis can indeed be effective in MICF we need to compare the radius of gyration of the charged pion in the magnetic field that separates the hot plasma from the cold plasma region (halo) with the dimensions of the region. Clearly, if the radius is larger than the thickness of the region or the radius of the system then muon catalysis, or for that matter, pion energy deposition as deduced above, will be of no value. The radius of gyration $(\mathrm{cm})$ of a particle of mass $m$ (amu), energy $E(\mathrm{keV})$ charge $Z$, situated in a magnetic field $B$ (tesla) is given by

$$
r_{L}=(0.45694) \frac{m^{2 / 2} E^{1 / 2}}{Z B}
$$

so that for a pion of mass $0.1493 \mathrm{amu}$, energy $236 \mathrm{MeV}$ in a magnetic field of 100 tesla, the result is $r_{L}=2.7123 \times 10^{-2}$ $\mathrm{cm}$ which is well within the dimensions of the pellet in MICF. Recalling from Eq. (17) that each negative pion decays (27ns decay time) into a muon which in turn gives rise to 150 fusion reactions we see that Eq. (15) must now be replaced by

$$
E_{f} \cong \frac{4}{3} \Pi R_{s}^{3}[\varepsilon+788](\mathrm{MeV})
$$

where the added energy reflects the alpha particle energy produced by 150 fusion reactions initiated by muons produced by 1.5 pions on the average and deposited in the plasma. Following the same reasoning as that presented after Eq. (15) we find that the amount of $\bar{H}$ required to supply $1.6 \mathrm{MJ}$ of energy that ignites the plasma is now given by $20.4 \mathrm{ng}$ and the amount needed to supply $1 \mathrm{MJ}$ is just $13 \mathrm{ng}$. In concluding this section it should be kept in mind that the long lifetime of the muons (i.e., $2.2 \mu \mathrm{s}$ ) is indeed more than adequate to initiate the burn in MICF since the burn time in this device is about $0.1 \mu \mathrm{s}^{6}$

It would be useful to compare the annihilation time $\tau_{a}$ with the heating time $\tau_{f}$ of the fusion fuel. In the interest of simplicity we assume that the heating in question is carried out only by the pions produced in the annihilation of the spark and the interaction is primarily with the electrons of the DT plasma which we take to be initially cold. The equation of interest in this case is ${ }^{6}$

$$
\frac{\mathrm{d} E}{\mathrm{~d} t}=-\dot{A} \frac{E}{T_{e}^{3 / 2}}
$$

where

$$
A=2 \times 10^{-12} n_{f} / m_{\pi}
$$

and $m_{\pi}$ is the pion mass ( $\left.=0.1493 \mathrm{amu}\right)$. Using the energy balance equation, namely

$$
\frac{3}{2} T_{e}=R\left(E_{0}-E\right)
$$

where $E_{0}$ is the initial pion energy $(236 \mathrm{MeV})$, and $R$ is the number of incident particles per electron of the DT plasma, we can substitute in Eq. (20) to get

$$
\frac{\mathrm{d} T_{e}}{\mathrm{~d} t}=A\left[\frac{2 \mathrm{RE}_{0}}{3 T_{e}^{3 / 2}}-\frac{1}{T_{e}^{\mathrm{i} / 2}}\right]
$$

This equation can be integrated readily to yield the heating time $\tau_{f}$. Using the equilibrium condition, i.e., $E=\left(\frac{3}{2}\right) T_{e}$ we obtain from Eq. (22)

$$
T_{e}=\frac{2}{3} \mathrm{RE}_{0} /(1+R)
$$


and with this the integration of Eq. (23) yields

$$
\begin{aligned}
\tau_{f} & =\frac{1}{A}\left[\frac{2}{3} \mathrm{RE}_{0}\right]^{3 / 2}\left\{\ln \left(\frac{\sqrt{1+R}+1}{\sqrt{1+R}-1}\right)\right. \\
& \left.-\frac{2}{3} \frac{1}{(1+R)^{3 / 2}}-\frac{2}{(1+R)^{1 / 2}}\right\} \\
\tau_{f} & =\frac{1}{A}\left[\frac{2}{3} E_{0}\right]^{3 / 2} f(R)=9.317 \times 10^{-5} f(R)
\end{aligned}
$$

If we choose $T_{f}=20 \mathrm{keV}$, then we see from Eq. (24) that $R$ $=1.27 \times 10^{-4}$ for which $f(R)=1.137 \times 10^{-5}$, and $\tau_{f} \cong 1$ ns. The energy exchange time between the electrons and the fusion ions at these densities is comparable to $\tau_{f}$ so that at the end of such time the ion energy will be approximately $1 / 2 T_{e}$ $=10 \mathrm{keV}$ which is the value we will consider in the applications. It is also interesting to note that $\tau_{f} \cong 5 \tau_{a}$, so that when an antihydrogen pellet of the appropriate size is employed to initiate ignition in the MICF DT plasma, over $90 \%$ of the antihydrogen in the sphere is annihilated in about $2 \tau_{a}$ $=0.4 \mathrm{~ns}$. This time is compatible with the heating of the fusion fuel and the burn time in MICF, which as we have noted is about $1 / 10 \mu \mathrm{s}$.

\section{Propulsive Capability of Antimatter Driven MICF}

Although not addressed in this calculation, it is safe to presume that sufficient energy from the gamma rays and the pions gets deposited in the halo region and the metal inner surface to cause both ionizing and heating in these regions. Both of these effects are sustained by the bremsstrahlung emanating from the hot plasma in the core. As noted earlier, the pressure buildup in the regions external to the core help retard the expansion of the hot plasma, thereby allowing it to produce more fusion energy. We have also seen that an input energy of $1.6 \mathrm{MJ}$ to the DT plasma by the antimatter annihilation results in heating it to $10 \mathrm{keV}$, and at a density of $5 \times 10^{21}$ in a radius of $0.25 \mathrm{~cm}$, the governing equations show that ignition takes place producing an energy gain of about 724. Success of MICF as an energy producer depends, however, on the existence of a strong magnetic field that retards the flow of heat from the hot plasma to the metal shell. We recall that such a field can be generated when a laser beam is used to ablate the plasma through the thermoelectric effect. Can such a field be created in an antimatterdriven MICF? Can the thermoelectric effect arise when matter antimatter interact and annihilate in the boundary layer described previously? We note that if the injected $\bar{H}$ pellet does not fully occupy the "spark" region then only a portion of this pellet establishes contact with the DT wall, and interaction takes place over this limited contact region. It can be postulated then that a DT plasma with sharp density and temperature gradients can be created in this focal spot much like the situation when a laser impinges on it. It is therefore not unreasonable to assume that a strong magnetic field can be generated with the use of antimatter as the driver although perhaps at the expense of partial annihilation of the injected pellet since total instant contact with the target is not likely in this case.

For utilization in a propulsion device we make use of the pellet design summarized in Table 1 . If such a pellet is housed in a combustion chamber with a radius of $100 \mathrm{~cm}$ and an opening of $2.50 \mathrm{~cm}$ which may be viewed as part of a magnetic nozzle with near-perfect efficiency, then by allowing the plasma (i.e., the charged particles), including that resulting from complete ionization of the metal shell, to adiabatically expand at the end of the fusion burn then escape through the nozzle, we can compute the exhaust time of such a "propellant." This time dictates in turn the maximum repetition rate allowed.
Table 1 Pellet design for propulsion

\begin{tabular}{ll}
\hline \hline Inner radius of solid fuel & $0.25 \mathrm{~cm}$ \\
Outer radius of solid fuel & $0.30 \mathrm{~cm}$ \\
Outer radius of metal shell & $0.547 \mathrm{~cm}$ \\
Fusion fuel & $\mathrm{DT}$ \\
Hot plasma core density & $5 \times 10^{21} \mathrm{~cm}^{-3}$ \\
Initial plasma core temperature & $10 \mathrm{keV}$ \\
Input energy from annihilation & $1.6 \mathrm{MJ}$ \\
Total input energy & $2.59 \mathrm{MJ}$ \\
Gain factor & 724 \\
Total pellet mass & $8.75 \mathrm{~g}$ \\
\hline \hline
\end{tabular}

Table 2 Propulsion parameters for an MICF system

\begin{tabular}{ll}
\hline \hline DT ion exhaust velocity & $375 \mathrm{~km} / \mathrm{s}$ \\
Metal ion exhaust velocity & $43.8 \mathrm{~km} / \mathrm{s}$ \\
Effective spec. impulse $I_{\mathrm{sp}}$ & $0.451 \times 10^{4} \mathrm{~s}$ \\
Repetition rate, $\omega$ & $\leq 15.39 \mathrm{~s}^{-1}$ \\
Total thrust $F$ & $0.172 \omega \mathrm{kN}$ \\
Jet power, $P_{\text {jet }}$ & $4.046 \omega \mathrm{MW}$ \\
\hline \hline
\end{tabular}

For the parameters listed above it can be shown that a rep rate $\omega=100$ is well within that limit and the corresponding propulsion parameters are summarized in Table 2 .

\section{Application to Mars Missions}

As noted earlier, we apply the results of the MICF propulsion consideration to a round trip from Earth to Mars. For a laser-driven system, we recall that the dry weight of the vehicle must include the power supply system for the laser and, at an input energy of $2.6 \mathrm{MJ}$, such a system including the driver (lasers, radiation, optics, etc.), the thrust chamber and other components is estimated ${ }^{12}$ to be 564 matric tons (MT). For the mission of interest we will consider a continuous burn, acceleration/deceleration trajectory profile which assumes constant $I_{\mathrm{sp}}, F$, and $P_{\text {jet }}$ operation. The equations that describe the transit time for the outbound and return legs of the journey, $\tau_{\mathrm{RT}}$, from Earth (e) to Mars (m) along with the distances traveled $D_{\mathrm{em}}$, and $D_{\mathrm{me}}$ are given by ${ }^{13}$

$$
\begin{gathered}
\tau_{\mathrm{RT}}=\tau_{\mathrm{em}}+\tau_{\mathrm{me}}=\frac{I_{\mathrm{sp}}}{F / W_{f}}\left(\frac{1}{\alpha \beta}-1\right) \\
D_{\mathrm{em}}=\frac{g I_{\mathrm{sp}}^{2}}{F / W_{f}}\left(\frac{1}{\beta}\right)\left(\frac{1}{\sqrt{\alpha}}-1\right)^{2} \\
D_{\mathrm{me}}=\frac{g I_{\mathrm{sp}}^{2}}{F / W_{f}}\left(\frac{1}{\sqrt{\beta}}-1\right)^{2} \\
\frac{1}{\alpha}=\frac{M_{i}}{M_{B}} ; \quad \frac{1}{\beta}=\frac{M_{B}}{M_{f}} ; \quad M_{B}=M_{f}+M_{p}^{B \rightarrow A}
\end{gathered}
$$

where $W_{f}=g M_{f}$ is the dry weight of the vehicle, $M_{i}$ is the initial mass, and $M_{p}$ is the propellant mass. Using the linear distance from Earth to Mars as $0.52 \mathrm{AU}$ (1 astronomical unit $=1.5 \times 10^{11} \mathrm{~m}$ ) we can calculate $\alpha$ and $\beta$ from Eqs. (27) and (28) which upon substitution in Eq. (26) yields the round trip time. For the laser-driven system it has been shown ${ }^{14}$ that such a trip takes 165 days when a rep rate $\omega=100$ is used and clearly longer if $\omega=10$.

If we now utilize the antimatter pellets as the driver then that portion of $W_{f}$ representing the laser driver can be eliminated giving us an $M_{f}=220 \mathrm{mT}$. At an $\omega=10 \mathrm{we}$ find that the trip takes 186 days, and at an $\omega=100$ it takes about 110 days. We recall from Sec. III that the amount of antihydrogen $(\vec{H})$ required to provide an input energy of $1.6 \mathrm{MJ}$ (needed to ignite the plasma) is $20.4 \mathrm{ng}$, and at $\omega=100$ the amount of $H$ needed for this mission is about $9.72 \mathrm{~g}$. For the slower trip, i.e., $\omega=10$, the amount of $\bar{H}$ needed is $1.73 \mathrm{~g}$. 
If we choose to use the $\mathrm{DH}_{\mathrm{e}}^{3}$ fuel cycle to minimize the neutron production, then the round-trip time using a laser driver increases to about 283 days ${ }^{15}$ due to the marked increase in laser input energy (and with it the increase in $W_{f}$ ) to accommodate the high $(\sim 50 \mathrm{keV})$ temperature needed to ignite the plasma. In this case the antimatter annihilation will be required to provide about $4 \mathrm{MJ}$, and for the same $W_{f}$ as in the DT case at $\omega=100$ we find that the trip time is reduced to about 213 days. In this case the amount of $\bar{H}$ needed will be about $38 \mathrm{~g}$.

\section{Conclusion}

Employing assumptions that have to be validated someday, we have demonstrated, in this investigation, the feasibility of using antimatter annihilation energy to drive a fusion propulsion system based on an inertial confinement concept that makes use of a very strong self-generated magnetic field. With these assumptions we have shown that modest amounts $(\because 10$ g) of antihydrogen will be needed to make a one-way trip to Mars in about 2 months if the technology allows us to make $\bar{H}$ ice pellets of minute radii that can be stored, manipulated, and fired at the rate of $100 / \mathrm{s}$. If the rep rate is reduced by a factor of 10 , we have shown that about $2 \mathrm{~g}$ will be needed but the trip will take nearly twice as long. If the $\mathrm{D}-\mathrm{H}_{\mathrm{e}}^{3}$ fuel cycle is employed to minimize the neutron production, this analysis shows that significantly larger amounts of $\vec{H}$ will be needed and the trip will take almost three times as long.

\section{Acknowledgmenit}

This work was supported in part by DOE and NASA.

\section{References}

${ }^{1}$ Kammash, T., and Galbraith, D. L., "A Promising Fusion Approach to Advanced Space Propulsion," Journal of British Interplanetary Society, Vol. 41, 1988, p. 527.
${ }^{2}$ Kammash, T., and Gạlbraith, D. L., "Mars Missions with the MICF Fusion Propulsion System," AIAA Paper 88-2926, July 1113, 1988.

${ }^{3}$ Kammash, T., and Galbraith, D. L. "Reaction Physics and Mission Capabilities of the Magnetically Insulated Inertial Confinement Fusion Reactor," Journal of Propulsion and Power, Vol. 6, No. 4, 1990 , p. 412.

${ }^{4}$ Coomes, E. P., and Dagle, J. E., "The Advantages of Nuclear Electric Propulsion Without the Concerns of Nuclear Power," Proceedings of 7th Symposium on Space Nuclear Power Systems, Albuquerque, NM, Jan. 7-10, 1990, p. 214.

5Cassenti, B., "Conceptual Designs for Antiproton Space Propulsion Systems," AIAA Paper 89-2333, July 1989 and references therein.

"Kammash, T., and Galbraith, D. L., "A High Gain Fusion Reactor Based on the Magnetically Insulated Inertial Confinement Fusion (MICF) Concept," Nuclear Fusion, Vol. 29, 1989, p. 1079.

${ }^{7}$ Kammash, T., Fusion Reactor Physics, Principles and Technology, Ann Arbor Science Publishers, Ann Arbor, MI, 1975, Chap. 4.

${ }^{8}$ Kammash, T., and Galbraith, D. L., "The Role of Fast Alphas in the Performance of MICF," Nuclear Fusion, Vol. 30, 1990, p. 1613.

${ }^{9}$ Kilcrease, D. P., and Kirkpatrick, R. C., "Magnetized Fuel Inertial Confinement Fusion," Nuclear Fusion, Vol. 28, 1988, p. 1465.

${ }^{10}$ Gsponer, V., and Hurni, J. P., "Antimatter Induced Fusion and Thermonuclear Explosions," Atomkernenergie-Kerntechnik, Vol. 49, 1987, p. 198.

${ }^{11}$ Jones, E. S., "Muon-Catalyzed Fusion Revisited," Nature, Vol. 321,1986, p. 127.

${ }^{12}$ Hyde, R. A., "A Laser Fusion Rocket for Interplanetary Propulsion," Lawrence Livermore National Laboratory Rept. UCRL$88857,1983$.

${ }^{13}$ Borowski, S. K., "A Comparison of Fusion/Antiproton Propulsion Systems for Interplanetary Travel," AIAA Paper 87-1814, June 1987.

${ }^{14}$ Kammash, T., "MICF - A High Gain Fusion Reactor for Power and Space Application," Proceedings of the 5th International Conference on Emerging Nuclear Energy Systems, ICENES '89, Karlsruhe, July 3-6, 1989 , p. 250.

${ }^{15}$ Kammash, T., and Galbraith, D. L., "Advanced Fuels for an Inertial Fusion Propulsion System," Proceedings of the 7th Symposium on Space Nuclear Power. Systems, Albuquerque, NM, Jan. 710,1990, p. 61 